\title{
Facilitators and Barriers to Breastfeeding Practices of Mothers in a Selected Urban Community in Malaysia
}

\author{
Naleena Devi Muniandy ${ }^{1,2^{*}}$, Fatin Iman Najihah Mohamad Yusof ${ }^{1}$ \\ ${ }^{1}$ Centre of Dietetics Study, Faculty of Health Sciences, Universiti Teknologi MARA, \\ Cawangan Selangor, 42300 Puncak Alam, Selangor, Malaysia \\ ${ }^{2}$ Maternal, Infant and Young Child Nutrition (MiChild) Research Group, Universiti Teknologi MARA, \\ Cawangan Selangor, 42300 Puncak Alam, Selangor, Malaysia
}

\begin{abstract}
This study was designed to explore the facilitators and barriers of breastfeeding practices among mothers in a selected urban population in Malaysia. This study reports the qualitative findings from a larger study that was conducted in two health clinics in Selangor, Malaysia. Ten mothers were recruited and an in-depth interview using grounded theory approach was used to obtain information. Interviews were transcribed and was manually arranged into codes and themes using thematic analysis. Facilitators to breastfeeding in this population were maternal factors (knowledge of breastfeeding, intention to breastfeed, perceived benefits of breastfeeding), social support and health care provider. Barriers to breastfeeding were insufficiency of breastmilk, employment and stigma of public breastfeeding. The findings of this study suggest that intervention to improve breastfeeding uptake should aim on increasing knowledge of mothers on coping with issues related to breastfeeding difficulties such as how to increase or sustain milk production and increasing awareness of employers and public on supporting breastfeeding mothers.
\end{abstract}

Keywords: breastfeeding, early life nutrition, infant nutrition, maternal experience, qualitative

\section{INTRODUCTION}

Breastfeeding supports healthy physical and cognitive development of a child (Wallenborn et al. 2021). Breastfeeding is also associated with development of food preferences, hunger regulation and promotes healthy mental health in a child (Verduci et al. 2014; Adan et al. 2019; Nguyen et al. 2021). Breastmilk contains bioactive compounds such as hormones, cytokines, immunoglobulins and other beneficials compounds that increases defence against harmful pathogens and increases tolerance towards antigens (Carr et al. 2021). Recent studies documented that breastfeeding protects against future obesity and non-communicable diseases such as hypertension, diabetes and atherosclerosis (Verduci et al. 2014; Binns 2016). Studies also show that breastfeeding helps to reduce the risk of ovarian cancer, breast cancer and important in spacing out child (Victoria et al. 2016).

The World Health Organization (WHO) has recommended that infants should be exclusively breastfed for the first six months and followed by introduction to solids from six months on words with the continuation of breastfeeding untill two years of age (WHO 2020). However, adhering to the recommendations still remains a challenge (United Nations Children's Fund (UNICEF) 2019).

Global report indicated that only $41 \%$ of infants under six months old are exclusively breastfed, $71 \%$ were breastfed at one year of age and only $45 \%$ were breastfed at two years of age worldwide (UNICEF/WHO 2017). In Malaysia, the National health and Morbidity Survey in 2016 reported that only $47.1 \%$ of the infants in this country were exclusively breastfed until six months while only $39.4 \%$ of the infants were breastfed untill two years old (National Health and Morbidity Survey 2016).

A mother's successful breastfeeding journey is determined by factors involving herself, her child and people around the mother. Maternal education, age, perception, experience, nature of birth and external factors such as family support, involvement of husband and grandmothers, peers and etc., play an important role in the success of breastfeeding among mothers (Emmott \&

\footnotetext{
"Corresponding Author: tel: +60332584383, email: naleena@uitm.edu.my

(Received 19-06-2021; Accepted 13-09-2021; Published 29-11-2021)
} 
Mace 2015; Martin et al. 2020; Theodorah \& Mc'Deline 2021). Studies show that mothers that receive support from their mothers, mothers in law, husband and friends tend to breastfeed longer compared to those who did not receive these support (Martin et al. 2020; Theodorah \& Mc'Deline 2021).

The external environment such as the involvement of healthcare practitioners, policies revolving breastfeeding and maternity leave, breastfeeding facilities in working premise and shopping mall play an important role in sustaining breastfeeding in mothers (Rollins \& Doherty, 2019). Studies show inconsistent findings on the level of education and support received from healthcare practitioners worldwide (Chang et al. 2020; (Fox et al. 2015). Some studies have reported an increase in breastfeeding prevalence after the constant education done during prenatal appointment while there are studies that have reported a poor initiation of breastfeeding even after childbirth despite having received prenatal education on breastfeeding (Mehlawat et al. 2020). Limited maternity leave and absence of breastfeeding facilities in workplace also remains a barrier to breastfeeding (Febrianingtyas et al. 2019).

A large pool of literature exists on the facilitators and barriers of breastfeeding globally. However, the way of how these factors influence mothers throughout breastfeeding differs according to population, country, degree of urbanization, religion and etc. Hence identifying which and how all these factors influence a mother's experience in breastfeeding in a population will help in planning effective interventions to encourage breastfeeding in the targeted population.

Hence, this study was designed to explore the predictors and barriers of breastfeeding practices among mothers in a selected urban population in Malaysia. The qualitative design of the study was able to identify issues that had been overlooked in a multi ethnic setting like Malaysia. The multi ethnic Asian platform was able to highlight cultural norms and obstacles faced by these mothers. The results, of this study may serve a guideline to understand the predictors and barriers of breastfeeding in this country and other multi ethnic population globally to improve existing policies and guidelines to improve prevalence of breastfeeding.

\section{METHODS}

\section{Design, location, and time}

The data from this study was obtained from a larger study that was conducted in 2019. The original study aimed to assess feeding practices and growth among the infants. It was a mixed methods study involving quantitative and qualitative design. In the quantitative part of the study, prospective data on infant feeding and growth were obtained while in the qualitative part, issues regarding infant feeding were obtained through in-depth interviews using a grounded theory approach. This study reports the qualitative findings from the bigger study.

The study was conducted in two community health clinics in Klang Valley Selangor. The two community health clinics were in Meru and Puncak Alam. The location was decided based on the availability of high number of mother infants' pairs from all the three ethnic groups that has been decided as the inclusion criteria of this study. One hundred fifty mother infant pairs were recruited in the larger study, in which ten mothers were enrolled for the qualitative part. This study reports the findings from the in-depth interviews.

A grounded theory approach was used to identify, analyse and develop a theoretical framework that describes predictors and barriers in breastfeeding practices among the study population (Glaser \& Strauss 2009). Mothers of the infants who agreed to join the qualitative part of the study were interviewed using an in-depth interview method. A semi structured interview guide was used to facilitate the interview to ensure information regarding the areas of interest were obtained.

Ethical approval for the original study was obtained from the National Medical Research Register (NMRR), reference No: KKM/NIHSEC/ PIT-811, prior to commencing any field work. Informed consent was read in either English or Malay, before obtaining participant's consent through a signature.

\section{Sampling}

All the ten participants included in this study were mothers of infants aged six to eleven months old. All their infants had a birth weight of $2,500 \mathrm{~g}$ to $4,000 \mathrm{~g}$ and were full term infants (37 to 42 gestation weeks) from singleton pregnancy. They were selected based on their willingness to 


\section{Predictors and barriers to breastfeeding}

participate in the in-depth interviews and also based on the emerging themes such as employed and unemployed mothers, mothers with lactation issues, mothers who consulted healthcare professionals and other immediate people to seek information and etc throughout data collection. Mothers whose infants were admitted for three days or more, two weeks before data collection or diagnosed with any congenital, medical or digestive problems that may impair their dietary intake were excluded.

Participants were given pseudonyms according to breastfeeding status at the point of data collection (NB for non-breastfeeding, and B for breastfeeding) and their ethnic group ( $\mathrm{M}$ for Malay mothers, I for Indian mothers and $\mathrm{C}$ for Chinese mothers) followed by hierarchy of point of contact (one for first mother interviewed, two for second mother interviewed etc).

\section{Data collection}

Participants whom the primary investigator or Co researcher identified as suitable to be recruited in the qualitative part of the study based on the emerging themes were approached. Participants were interviewed by either the Primary investigator or Co-researcher who were females, only if they agreed to be involved in the qualitative part of the study. These participants were interviewed in either English or Bahasa Melayu (national language) using an in-depth interview method guided by a semi structured interview guide.

The semi structured interview guide was designed to enable us to capture the various facilitators and barriers to breastfeeding. The questions were designed to explore conditions that may affect breastfeeding practices that may promote or obstruct mothers from breastfeeding. These questions were initially informed by the literature and included: (a) Duration of breastfeeding and non-breastfeeding practices; (b) Knowledge seeking on breastfeeding; (c) Reasons for quitting or sustaining breastfeeding; (d) Support received on breastfeeding. Along the way, probes and extra questions were added guided by a grounded theory approach.

Participants were approached and interviewed till data saturation was reached, in which there were no newer codes or themes that emerged from the interviews. Each in depth interview lasted for 30 minutes to an hour.
Observations were recorded as field notes in a journal throughout the interviews.

\section{Data analysis}

The audio interviews were transcribed verbatim. Interviews were translated to English language and sections from the transcripts that were unclear were checked back with the audio recording. Data was sorted and coded manually. The interviews were first coded by the Co researcher and later was independently read and coded by the principal investigator. Codes were arranged into themes guided by a thematic analysis approach (Braun \& Clarke 2006).

\section{RESULTS AND DISCUSSION}

\section{Maternal sociodemographic and prenatal characteristics}

A total of ten mother infant pairs were involved in this study. Most of the mothers were between 30 to 34 years old. Mothers were largely from the Malay ethnic group $(n=7)$ while the remaining $(n=3)$ were from the non-Malay ethnic group. Most mothers, $(n=7)$ had a secondary school education while only one of them had a primary school education while the remaining two mothers had a tertiary school education. Majority of the participants, $(n=7)$ reported a household monthly income of more than USD719 (RM3,000) while only one participant reported an income of less than USD239.5 (RM1,000). The study also indicated that Most mothers $(n=6)$ worked after delivery. The participants' characteristics are shown in Table 1.

Most mothers in this study ( $\mathrm{n}=6)$, had a normal pre-pregnancy Body Mass Index (BMI), $\left(18.5-24.9 \mathrm{~kg} / \mathrm{m}^{2}\right)$. The study indicated that $(n=5)$ of the mothers fell 'under recommended weight gain' group, $(n=3)$ of the mothers fell within a "recommended weight gain" category, while $(n=2)$ of the participants fell in the "over the recommended weight gain" group. Maternal prenatal factors are included in Table 1.

\section{Infant characteristics}

There were six male infants and four female infants involved in this study. Most infants were not the first child $(\mathrm{n}=7)$ and mothers reported that all of them had breastfed their child. However only $(n=6)$ of the infants were exclusively breastfed till six months. Three of them were 
Table 1. Maternal sociodemographic and prenatal characteristics

\begin{tabular}{cc}
\hline \multicolumn{1}{c}{ Variables } & Frequency $(\mathrm{n})$ \\
\hline Age (years) & 2 \\
$25-29$ & 6 \\
$30-34$ & 2 \\
35 and above & \\
$(32.10 \pm 3.178)$ &
\end{tabular}

Ethnicity

Malay

Non-Malay

Education level

Primary school

Secondary school

Tertiary level

Household income

$<239.5$ USD

(RM1,000)

239.5-718.5USD

(RM1,000-RM2,999)

$\geq 719$ USD

(RM3,000)

Work after delivery

Yes

No

Pre-pregnancy BMI $\left(\mathrm{kg} / \mathrm{m}^{2}\right)$

18.5-24.9

25.0-29.9

$\geq 30.0$

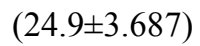

Gestational weight gain

Under recommended weight gain

Within recommended weight gain

Over the recommended weight gain

$(6.03 \pm 6.65)$

RM; Ringgit Malaysia; USD: United States Dollar; BMI: Body Mass Index introduced to solids before six months of age. Infants' characteristics are shown in Table 2.

\section{Facilitators and bbarriers to breastfeeding} among mothers in this study

The results on the main themes and subthemes that emerged on the predictors and facilitators of breastfeeding are given in Figure 1. Three major themes emerged on the area of predictors for breastfeeding among the participants: maternal factors, social support and healthcare provider while the themes that emerged on the area of barriers of breastfeeding perceived were lactation problem, employment, and stigma of public breastfeeding. The model developed from the themes and codes obtained from this study is shown in Figure 1.

\section{Facilitators to breastfeeding \\ Maternal factors}

Knowledge of breastfeeding. Knowledge on breastfeeding emerged as a subtheme for predictors of breastfeeding. Mothers in this study were aware of the importance of breastfeeding to their infants. A common importance of breastfeeding that they were aware of was the importance of breastfeeding to protect the child from common illness such as flu, fever and other diseases. This knowledge, was a motivator for some of the mothers to breastfeed their infants.

"I breastfeed my baby until six months. I planned to breastfeed my baby because I know breastmilk is very nutritious and could protect my baby from getting a fever, flu, and other diseases". BFM1.

Intention to breastfeed. An important predictor for breastfeeding in this study was having an intention to breastfed from pregnancy. The interviews suggested that mothers who had exclusively breastfeed their infants until 6 months and plan to continue breastfeeding until two years of age, claimed to have planned this since they were pregnant. This was apparent in the conversation of two mothers:

"Yes, I planned to breastfeed my baby since I was pregnant. I planned to continue to breastfeed until two years". I'm able to give my breastmilk to my baby up to eight months now and I know breastmilk is much better for my baby's growth". BFM3.

This study found that maternal factors such as perceived benefits, knowledge and 


\section{Predictors and barriers to breastfeeding}

Table 2. Infant's characteristics

\begin{tabular}{|c|c|}
\hline Variables & $\begin{array}{l}\text { Frequency } \\
\quad(\mathrm{n}=10) \\
\text { Mean } \pm \text { SD }\end{array}$ \\
\hline \multicolumn{2}{|l|}{ Gender } \\
\hline Boy & 6 \\
\hline Girl & 4 \\
\hline \multicolumn{2}{|l|}{ Hierarchy in siblings } \\
\hline First child & 3 \\
\hline Second and above & 7 \\
\hline \multicolumn{2}{|l|}{ Ever breastfed (0-24 months) } \\
\hline Yes & 10 \\
\hline No & - \\
\hline \multicolumn{2}{|l|}{$\begin{array}{l}\text { Continued breastfeeding } \\
\text { (after } 6 \text { months) }\end{array}$} \\
\hline Yes & 6 \\
\hline No & 4 \\
\hline \multicolumn{2}{|l|}{$\begin{array}{l}\text { Exclusively breastfeeding } \\
(0-6 \text { months })\end{array}$} \\
\hline No & 3 \\
\hline Yes & 6 \\
\hline Mixed feeding & 1 \\
\hline \multicolumn{2}{|l|}{$\begin{array}{l}\text { Introduction to formula feeding } \\
(\mathrm{n}=9)\end{array}$} \\
\hline Yes & 6 \\
\hline No & 3 \\
\hline $\begin{array}{l}\text { Age introduction to formula } \\
\text { feeding }(0-24 \text { months })(n=9)\end{array}$ & $6.6 \pm 5.0$ \\
\hline Never & 3 \\
\hline$<6$ months & 3 \\
\hline At 6 months and above & 3 \\
\hline $\begin{array}{l}\text { Age Introduction to solid foods } \\
\text { (at } 6 \text { months) }\end{array}$ & $6.9 \pm 0.74$ \\
\hline$<6$ months & 1 \\
\hline 6 months and above & 9 \\
\hline
\end{tabular}

intention to breastfeed are important predictors to breastfeeding. A study conducted among breastfeeding mothers in Kelantan, Malaysia reported that those with prior experience in breastfeeding and high scores in knowledge of breastfeeding had a higher odds of exclusively breastfeeding their infant at one week postpartum
(Che'Muda et al. 2018). A meta-analysis also stated that maternal knowledge is associated with breastfeeding at one year postpartum (ZakarijaGrković et al. 2016). However, maternal intention to breastfeed has been shown to be a strong predictor to breastfeed regardless of knowledge or experience suggesting that mothers need to be given knowledge of breastfeeding during pregnancy to encourage them to have the intention to sustain breastfeeding for two years (Raissian \& Su 2018).

Perceived benefits of breastfeeding. One of the facilitators of breastfeeding in this study was the perceived benefits of breastfeeding by mothers. In this study, mothers were aware that breastmilk is nutritious and helps to increase the immunity of the infants. This was apparent in the conversation of a mother who claimed that breastmilk was a nutritious food and helped to protect her infant from illness:

"In my opinion, babies who consumed breastmilk were healthier and rarely get flu, fever and other diseases". BFM1.

Mothers also revealed that breastfeeding was easier and more convenient than formula feeding. Breastmilk was also believed to be healthier than formula milk that was claimed to contain more sugar. In addition, mothers who participated in this study also associated benefits of breastfeeding with an advantage of reducing household expenses. Participants claimed that breastfeeding was cost effective as breastmilk was free while formula milks were expensive. This was evident in the conversation with a mother:

"Breastfeeding can save money as formula milk is very expensive, and formula milk contained more sugar compared to breast milk". BFM7.

Mothers in this study perceived breastmilk as a healthier choice compared to formula milk as it does not contain high sugar and helps to increase the immunity of their child. They also state that breastfeeding is easier, convenient and cost effective. These factors were associated with benefits of breastfeeding hence a facilitator to breastfeed among the mothers.

\section{Social support}

Social support plays an important role as a predictor to breastfeeding in this study. Husbands, family members, and friends were important factors that influence mothers to successfully 


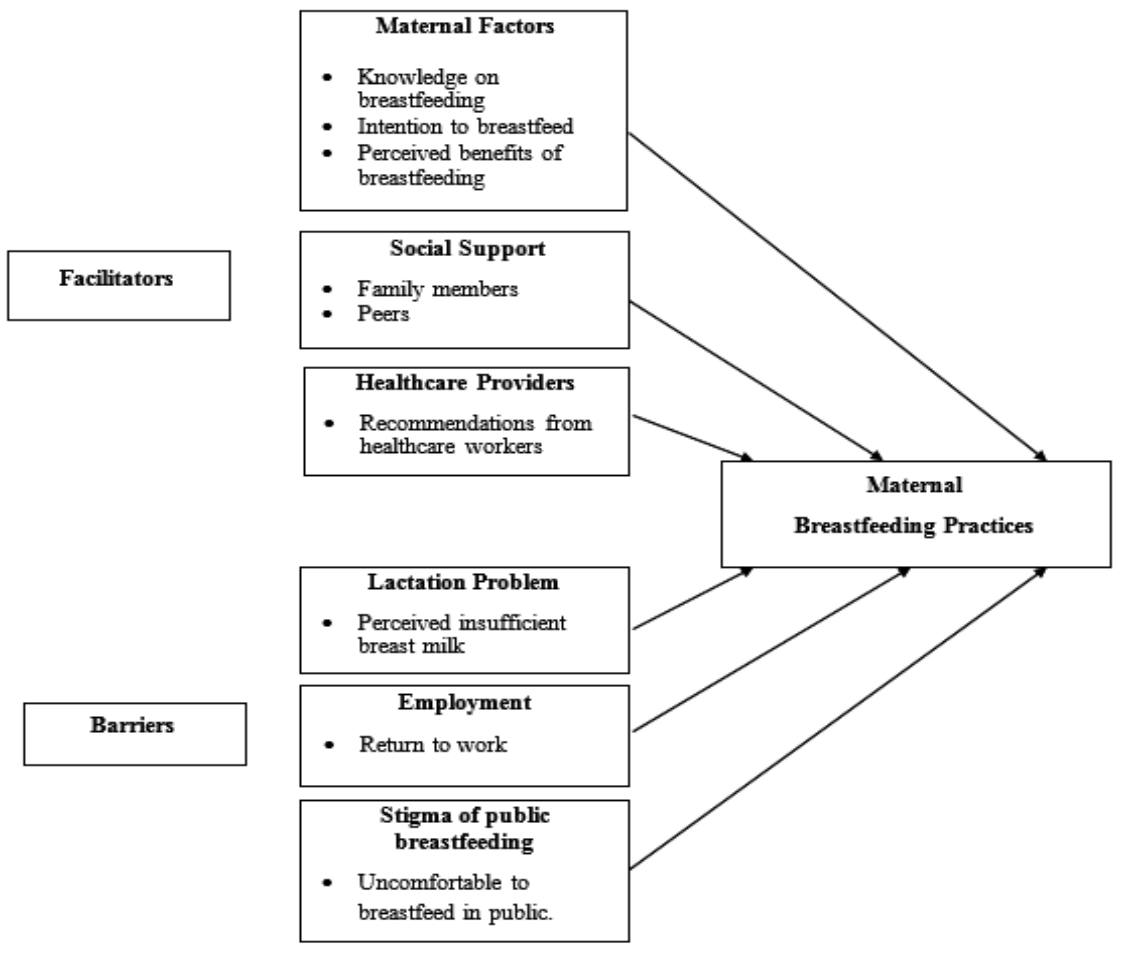

Figure 1. Model for facilitators and barriers to maternal breastfeeding practices in a selected urban community in Malaysia.

breastfeed. Most participants claimed that they learned about breastfeeding from their family members, especially their mothers and also friends. Conversation with an Indian mother revealed the encouragement given by her mother to continue breastfeeding by associating the importance of breast milk as a "gift from god".

"My mom encouraged me to continue to breastfeed my baby, and, when I stopped breastfeeding, my mother scolded me as she said that breastmilk is a gift from God". BFI3.

Peer support too emerged as an essential factor that played a role as a predictor for breastfeeding. Exchanging problems and solutions among friends who are also breastfeeding was important in the breastfeeding journey. This was narrated by one of the mothers:

"Oh, I learned a lot about breastfeeding from my friends. It is much convenient to ask my friends because most of my friends have a baby around the same age as mine. We always ask for each other's opinion". BFM4.

The role of a husband is very important in helping a mum to sustain her breastfeeding journey. Most mothers in this study expressed that they received good support from their husband to breastfeed. One of the mother, UM1 explained that her husband use to massage her back after she breastfeeds her baby. This finding further highlights that husbands were very supportive in encouraging their wives to breastfeed.

In this study, the immediate people around a mother, such as grandmothers, husband and friends were important factor for a successful breastfeeding journey among the participants. Mothers need to be comfortable, and have a support system in which they can communicate their problems and ask for suggestions from other. Having someone who is approachable and have experience in the area of breastfeeding kept the mothers motivated to continue their breastfeeding practices.

Studies show that support from family members, relatives, husband and peers may determine the success or failure of breastfeeding among the mothers (Martin et al. 2020). This depends on how supportive or discouraging this inner circle of people are to the mothers. Although many of them are aware of the importance of breastfeeding and do advice on how to cope with an issue during breastfeeding, studies show that there are some of them who would encourage 


\section{Predictors and barriers to breastfeeding}

formula feeding or to introduce complementary feeding before six months (Theodorah \& Mc’Deline 2021).

Husband or father of the child can be a barrier or support to breastfeeding. In developing countries, presence of husband is associated with early termination of breastfeeding while in developed countries a mother may not be successful in breastfeeding her child for a period of six months without the support of her husband (Emmott \& Mace 2015). This shows that the perception on breastfeeding varies according to population or location.

\section{Healthcare provider}

Healthcare providers, such as doctors and nurses, played an important role as predictors to breastfeeding among the mothers in this study. All the mothers involved in this interview narrated that they have been educated on the importance of breastfeeding. They were also taught on how to breastfeed during their pregnancy. Although the interviews reported the involvement of healthcare team in educating the mothers on breastfeeding, but the role of nurses were highlighted in most of the conversation with the mothers. Interview revealed that mothers were taught on breastfeeding in a seminar during their pregnancy:

"I learned about breastfeeding from my family members and nurses, and I did attend the seminar on breastfeeding. The nurses taught us how to breastfeed in that seminar". BI1.

In certain cases, the nurses were very encouraging on breastfeeding. One mother revealed that she was encouraged by one of the nurses to breastfed her child within the first hour, just after her childbirth:

"On the first day of my delivery, I had started to breastfeed him because the hospital staff encouraged me to start breastfeed within the first hour of my baby's life". BFI3.

The important role played by the healthcare providers either at the community health clinics or at the hospitals, just after childbirth is important as a reminder and precursor for breastfeeding for these mothers to breastfeed. A study on women's experience in breastfeeding in the United Kingdom reported a mixed response towards the involvement on healthcare providers in their breastfeeding experience. Mothers complained that healthcare providers did not elaborate on the challenges and difficulty of breastfeeding resulting in mothers were unprepared and stressed after their delivery. The prolonged challenges and frustration of not being able to meet the demand of their breastfeeding child resulted in them to quit breastfeeding (Fox et al. 2015).

\section{Barriers to breastfeeding Lactation problem}

Perceived insufficient breastmilk was a common barrier for mothers to exclusively breastfeed for the first six months or to continue breastfeeding until two years. They were four women in this study that claimed they stopped breastfeeding because they did not have sufficient breastmilk.

"I don't breastfeed my baby anymore... the longest period I breastfeed my previous baby was up to 10 months, but for this baby, I have stopped at four months... I realized that I just don't have breastmilk and it's just too little.", stated another mother. NBFM6.

The findings also revealed that, mothers did try to express their breastmilk to breastmilk their infant, however this method also was not successful hence resulting them to switch to formula milks.

"I had to stop breastfeeding my child when he was eight months. Because I realize that my milk was insufficient. And if I pump, I was only able to get one oz only", said one mother. NBFM5.

Perceived insufficient breastmilk is a common problem linked with early caseation of breastfeeding. In this study, this factor emerged as a barrier to breastfeeding. However, the point that mothers did try to express their breastmilk via a pump suggest that they tried their best to provide breastmilk to their child, but the fact that they still could not yield enough milk made them to stop breastfeeding.

Perceived insufficient breastmilk had always been a reason for early termination of breastfeeding. A recent study in Taiwan, involving 1,000 participants reported that nearly $40 \%$ of the mothers stopped exclusively breastfeeding at one month postpartum as they thought they could not produce sufficient milk (Chang et al. 2019). Previous study also reported a consistent findings in which perceived insufficient breastmilk was among the top three reason for early cessation of breastfeeding (Wagner et al. 2013). Addressing 
maternal concern on insufficient breastmilk may help to reduce the tendency to stop breastfeeding early in life.

\section{Employment}

Employment emerged as an important barrier to breastfeed among the participants. In this study, Mothers claimed they did not have enough time to express their breastmilk to be given to their infants during working hours. In addition, working resulted in them being tired hence they were not able to get up in the night to express their breastmilk, thus resulting them to stop breastfeeding and introduce formula milk to their infants.

"I stop breastfeeding her after six months. I don't have enough time to express my breastmilk for her when I started to work, as I feel tired". NBF12.

Mothers also claimed that their babies refused to be breastfed directly after they were given breastmilk via bottle. This was another reason, for mothers to stop breastfeeding as they claimed they were too tired to pump their breastmilk at night as they were tired after work. This slowly resulted in them to introduce formula milk to their infants as they found it more convenient. In addition, there were mothers who complained that their breastmilk production reduced drastically after they started working, resulting them to stop giving breastmilk.

Breastfeeding mothers often quit breastfeeding after resuming work, this finding has been consistent in many studies. However, increasing the length of paid maternity leave does promote breastfeeding in low- and middleincome countries (Chai et al. 2018). Employed mothers also reported feeling tired and sleep deprived and the challenge of finding time to pump milk during working hours too resulted in poor milk production which eventually leads to termination of breastfeeding (Chang et al. 2019). Break time in between working hours may be helpful to support breastfeeding mothers to find time to express their breastmilk.

\section{Stigma of public breastfeeding}

Mothers still feel uncomfortable to breastfeed in public in this $21^{\text {st }}$ century. Stigma of public breastfeeding exist in this population resulting the mothers to find a closed and private place to breasted. Some chose to breastfeed in the car before heading to their destinations with their infants.

"Of course, I feel shy to breastfeed in public. I will breastfeed my baby in the car before going out", NBFI2 mentioned one mother.

It is clear that although breastfeeding is encouraged by family members, healthcare providers and is a normal practice of feeding a child, but stigma of breastfeeding still exist in the studied population. The inconvenience of not being able to breastfeed anytime at any location resulted in the mothers to opt for formula milk to feed their child in public.

A review on breastfeeding among overweight and obese mothers found that these group of mothers are embarrassed to breastfeed in public because of the fear of exposing their body to others (Chang et al. 2020). A study in Australia among breastfeeding mothers also reported that mothers often feel uncomfortable to breastfeed in public as they constantly need to avoid male gazing at them or people coming to see the baby while they are breastfeeding (Sheehan et al. 2019). Hence, they often cover up and this led to them feeling scared of suffocating their child or afraid that their child is not being fed properly. This discomfort remains a barrier in many countries in which social norm include them to cover themselves up properly if they breastfeed in public (Coomson \& Aryeetey 2018; Sheehan et al. 2019). It is important to create awareness among public and breastfeeding mothers in order to provide a comfortable environment for mothers to breastfeed to support breastfeeding.

\section{CONCLUSION}

Many guidelines and policies exist on breastfeeding suggesting that people are aware of the importance of breastfeeding globally. However, facilitators and barriers to breastfeeding differ according to population. This study suggests that maternal perceived insufficient breastmilk, returning to work and being ashamed to breastfeed in public as main barriers to breastfeed in the studied population. Hence, intervention should aim on increasing knowledge of mothers on coping with issues related to breastfeeding difficulties such as how to increase or sustain milk production and increasing awareness of employers and public on supporting breastfeeding mothers. 


\section{ACKNOWLEDGEMENT}

We are grateful to all the women who participated in this study.

\section{DECLARATION OF INTERESTS}

The authors declare that they have no conflicts of interest.

\section{REFERENCES}

Adan RA, van der Beek EM, Buitelaar J K, Cryan JF, Hebebrand J, Higgs S, Schellekens H, Dickson SL. 2019. Nutritional psychiatry: Towards improving mental health by what you eat. Eur Neuropsychopharmacol 29(12):1321-1332. https://doi. org/10.1016/j.euroneuro.2019.10.011

Binns C, Lee M, Low WY. 2016. The long-term public health benefits of breastfeeding. Asia Pac J Public Health 28(1):7-14. https:// doi.org/10.1177/1010539515624964

Braun V, Clarke V. 2006. Using thematic analysis in psychology. Qual Res Psychol 3(2):77-101. https://doi. org/10.1191/1478088706qp063oa

Carr LE, Elolimy A, Rosa F, Virmani MD, Munblit D, Yeruva L. 2021. Role of human milk bioactives on infants' gut and immune health. Front Immunol 12:290. https://doi. org/10.3389/fimmu.2021.604080

Chai Y, Nandi A, Heymann J. 2018. Does extending the duration of legislated paid maternity leave improve breastfeeding practices? Evidence from 38 low-income and middle-income countries. BMJ Global Health 3(5):e001032. https://doi. org/10.1136/bmjgh-2018-001032

Chang PC, Li SF, Yang HY, Wang LC, Weng CY, Chen KF, Chen W, Fan SY. 2019. Factors associated with cessation of exclusive breastfeeding at 1 and 2 months postpartum in Taiwan Int Breastfeed J 14(1):18. https:// doi.org/10.1186/s13006-019-0213-1

Chang YS, Glaria AA, Davie P, Beake S, Bick D. 2020. Breastfeeding experiences and support for women who are overweight or obese: A mixed-methods systematic review. Matern Child Nutr 16(1):e12865. https://doi.org/10.1111/men.12865
Che'Muda CM, Ismail TAT, Ab Jalil R, Hairon SM, Sulaiman Z, Johar N. 2018. Maternal factors associated with the initiation of exclusive breastfeeding among mothers at one week after delivery in two selected hospitals in Kelantan, Malaysia. Malays J Med Sci 25(4):112-121. https://doi. org $/ 10.21315 / \mathrm{mjms} 2018.25 .4 .11$

Coomson JB, Aryeetey R. 2018. Perception and practice of breastfeeding in public in an urban community in Accra, Ghana. Int Breastfeed J 13(1):1-10. https://doi. org/10.1186/s13006-018-0161-1

Emmott EH, Mace R . 2015. Practical support from fathers and grandmothers is associated with lower levels of breastfeeding in the UK millennium cohort study. Plos One 10(7):e0133547. https://doi.org/10.1371/ journal.pone. 0133547

Febrianingtyas Y, Februhartanty J , Hadihardjono DN. 2019. Workplace support and exclusive breastfeeding practice: A qualitative study in Jakarta, Indonesia. Malays J Nutr 25(1):129-142. https://doi. org/10.31246/mjn-2018-0107

Fox R, McMullen S, Newburn M. 2015. UK women's experiences of breastfeeding and additional breastfeeding support: A qualitative study of baby café services. BMC Pregnancy and Childbirth 15(1):1-12. https://doi.org/10.1186/ s12884-015-0581-5

Glaser BG, Strauss AL. 2009. The Discovery of Grounded Theory: Strategies for Qualitative Research. New Brunswick (USA): Aldine.

Martin SL, McCann JK, Gascoigne E, Allotey D, Fundira D, Dickin KL. 2020. Mixedmethods systematic review of behavioral interventions in low- and middle-income countries to increase family support for maternal, infant, and young child nutrition during the first 1000 days. Current Developments in Nutrition 4(6):nzaa085. https://doi.org/10.1093/cdn/nzaa085

Mehlawat U, Puri S, Rekhi TK. 2020. Breastfeeding practices among mothers at birth and at 6 months in urban areas of Delhi-Ncr, India. J Gizi Pangan. 15(2):101-108. https://doi.org/10.25182/ jgp.2020.15.2.101-108 
National Health and Morbidity Survey. 2016. Maternal child health findings. Volume two: Maternal and child health findings. Institute for Republic Health, National Institute of Health, Ministry of Health Malaysia. http://iku.moh.gov.my/ images/IKU/Document/REPORT/2016/ N H M S 2016 R e p or t Vo 1 u m e I I MaternalChildHealthFindingsv2.pdf [Accessed 6th July 2020].

Nguyen AN, van Langeveld AW, de Vries JH M, Ikram MA, de Graaf C, Mars M, Voortman T. 2021. Dietary taste patterns in early childhood: The generation $r$ study. Am J Clin Nutr 113(1):63-69. https://doi. org/10.1093/ajen/nqaa296

Raissian KM, Su JH. 2018. The best of intentions: Prenatal breastfeeding intentions and infant health. SSM-Population Health 5:86-100. https://doi.org/10.1016/j. ssmph.2018.05.002

Sheehan A, Gribble K, Schmied V. 2019. It's okay to breastfeed in public but.... . Int Breastfeed J 14(1):1-11. https://doi. org/10.1186/s13006-019-0216-y

Theodorah DZ, Mc'Deline RN. 2021."The kind of support that matters to exclusive breastfeeding" a qualitative study. BMC Pregnancy and Childbirth 21(1):1-8. https://doi.org/10.1186/s12884-021 03590-2

[UNICEF] United Nations Children's Fund. 2019. Infant and young child feeding. Adopting optimal feeding practices is fundamental to a child's survival, growth and development, but too few children benefit. UNICEF data. https://data. unicef.org/topic/nutrition/infant-andyoung-child-feeding/ [Accessed 15 April 2021].
[UNICEF/WHO] United Nations Children's Fund/World Health Organization. 2017. Global Breastfeeding Scorecard 2017: Tracking progress for brestfeeding policies and programmes. https://www.who.int/ nutrition/publications/infantfeeding/ global-bf-scorecard-2017.pdf [Accessed 14th April 2021].

Verduci E, Banderali G, Barberi S, Radaelli G, Lops A, Betti F, Riva E, Giovannini M. 2014. Epigenetic effects of human breast milk. Nutrients 6(4):1711-1724. https:// doi.org/10.3390/nu6041711

Victora CG, Bahl R, Barros AJD, França GVA, Horton S, Krasevec J, Murch S, Sankar MJ, Walker N, Rollins NC. 2016. Breastfeeding in the $21^{\text {st }}$ century: Epidemiology, mechanisms, and lifelong effect. Lancet 387(10017):475-490. https://doi. org/10.1016/S0140-6736(15)01024-7

[WHO] World Health Organization. 2020. Infant and young child feeding. https://www. who.int/news-room/fact-sheets/detail/ infant-and-young-child-feeding [Accessed 15th April 2021].

Wagner EA, Chantry CJ, Dewey KG, NommsenRivers LA. 2013. Breastfeeding concerns at 3 and 7 days postpartum and feeding status at 2 Months. Pediatrics 132(4):e865-e875. https://doi.org/10.1542/peds.2013-0724

Wallenborn JT, Levine GA, Dos Santos AC, Grisi S, Brentani A, Fink G. 2021. Breastfeeding, physical growth, and cognitive development. Pediatrics 147(5). https://doi.org/10.1542/peds.2020-008029

Zakarija-Grković I, Šegvić O, Vučković Vukušić A, Lozančić T, Božinović T, Ćuže A, Burmaz T. 2016. Predictors of suboptimal breastfeeding: An opportunity for public health interventions. Eur J Public Health 26(2):282-289. https://doi.org/10.1093/ eurpub/ckv203 